\title{
Endometriosis: an enigmatic disease with many faces
}

\author{
Dipak J. Limbachiya, Grishma P. Agrawal*
}

\author{
Department of Obstetrics and Gynecology, EVA Endoscopy Training Institute, Ahmedabad, Gujarat, India
}

Received: 21 December 2016

Accepted: 13 December 2016

\author{
*Correspondence: \\ Dr. Grishma P. Agrawal, \\ E-mail: grishma.agrawal29@gmail.com
}

Copyright: (C) the author(s), publisher and licensee Medip Academy. This is an open-access article distributed under the terms of the Creative Commons Attribution Non-Commercial License, which permits unrestricted non-commercial use, distribution, and reproduction in any medium, provided the original work is properly cited.

\begin{abstract}
Endometriosis is a benign disease defined by the presence of the endometrial glands and stroma outside of the uterus, both at pelvic and extra pelvic sites. We are reporting 4 unusual cases of endometriosis that are unique on their own due to site of endometriosis, presenting symptoms, complications and treatment. Endometriosis was present at post caesarean scar site in one case, at vault site in another case, in bladder in third case and in the last case it caused frozen pelvis with hydroureter, hydronephrosis and possibly infertility. All four patients have been treated laparoscopically and have been following up with no recurrence of its symptoms. The purpose of this article is to create awareness among Doctors about endometriosis, which is more common than previously thought as. Endometriosis has been rightly summed up in the phrase "The clock is definitely ticking, as we know millions of women still live lives awash in anguish, just as they did thousands of years ago and just as they will centuries from now unless we steer ourselves faster toward the long-elusive cure. Four thousand years is long enough; the time has come to end the empire of endometriosis".
\end{abstract}

Keywords: Bladder endometriosis, Endometriosis, Hydroureter, Infertility, Laparoscopy, Post caesarean scar, Vault endometriosis

\section{INTRODUCTION}

Endometriosis is a benign disease defined by the presence of the endometrial glands and stroma outside of the uterus, both at pelvic and extra pelvic sites, first believed to be described by Rokitansky in $1860 .{ }^{1}$ The most commonly affected areas are the ovaries followed by the pouch of Douglas, uterosacral ligament (especially its insertion into the back side of the uterus), vesicouterine pouch, serosal surface of the uterus, fallopian tubes, round ligament, and rectovaginal septum. Endometriosis can also be located inside the genital tract and can spread into the cervix and vagina, especially the posterior vaginal wall, which is related to the frequently affected rectovaginal septum and in the perineum (especially on scars of previous episiotomies) or in the Bartholin gland. ${ }^{2}$ Extrapelvic endometriosis can be found in unusual places like in the nervous system, thorax, urinary tract, gastrointestinal tract, and in cutaneous tissues and its most frequent location is the abdominal wall. ${ }^{3}$ Malignant transformation of endometriosis may occur in up to $1 \%$ of women, with the most common site being the ovary. ${ }^{4}$ The main cause of extrapelvic implants is obstetric and gynecological procedures such caesarean section, hysterectomy, and it can even occur after laparoscopic surgery. ${ }^{5}$

The prevalence of asymptomatic endometriosis is $1-7 \%$ in women seeking elective sterilization, 12-32\% among women of reproductive age with pelvic pain, $9-50 \%$ in infertile women, and approximately $50 \%$ of teens with chronic pelvic pain or dysmenorrhea. ${ }^{6}$ Extra pelvic sites endometriomas are often confused with granuloma, hematoma, keloid, incisional hernia, vascular formation, sebaceous cyst, lipoma, or tumors and so patients often report general physicians, surgeons, or dermatologist because of atypical presentation and sometimes resulting 
in delay in diagnosis. ${ }^{7} \mathrm{We}$ are reporting four such unusual cases of endometriosis hoping to increase awareness of this rare condition.

\section{CASE REPORT}

\section{Case 1}

A 38 year old female patient, P1L1 presented in January 2015 with complain of painful swelling on the left side of the caesarean section scar. She had first noticed the pea sized swelling 1.5 year back, which gradually increased to the present size over the span of 1.5 years and became increasingly painful. The pain that was like a dull ache was more during the time of menses. She also had cyclical mild bleeding from the lesion at the time of menses since last 6 months. There was no history of bowel and bladder complains. She had undergone caesarean section 13 years back. She had history of hypertension detected 1 year back and was on antihypertensives. She was newly diagnosed as diabetic in last month. On examination, her abdomen appeared normal with pfannensteil scar of previous caesarean section. There was a $3 \times 4 \mathrm{~cm}$ lump at left angle of caesarean scar, round with smooth surface and irregular outline, firm consistency, tender and painful, and not fixed to rectus sheath. Ultrasound examination revealed hypoechoic lesion just below the external scar with no connection to the underlying muscle. The typical history, symptoms and presentation tilted our diagnosis towards endometriosis.

Patient was taken for Laparoscopic guided excision of the endometrioma lesion. Intra-operatively, uterus, ovaries, fallopian tubes were found to be normal with no evidence of other endometriotic patches or recto-vaginal nodule. Peritoneum along scar endometriotic nodule was identified and incised with no extension of nodule beyond rectus muscle. Decision to excise the endometrioma per abdomen was taken. Skin all around the endometrioma lesion taking $2 \times 2 \mathrm{~cm}$ of rectus sheath to obtain $2 \mathrm{~cm}$ of healthy margin was excised. Histopathological report confirmed diagnosis of endometriosis in skin and subcutaneous tissue with muscle being free of lesion.

\section{Case 2}

A 40 year old female P2L2 with history of Vaginal Hysterectomy (VH) done 3 years back for menorrhagia, came with complains of Post-coital bleeding, spotting PV, and abdominal pain on and off since 2 years. There was no history of bowel and bladder complains. The Vaginal Hysterectomy was conducted by an open technique in outside setup and the vaginal vault was sutured using a 1-0 vicryl. After one year in the postoperative period she started having above complains. She then underwent cauterization twice in one year but was not relieved of her complains. On per speculum examination there was bleed ++ from vault. Per vaginally it showed $2 \times 2 \mathrm{~cm}$ tender lesion more on posterior fornix. In differential diagnosis the possibility of vault granuloma, vaginal adenosis, malignancy and endometriosis were considered. Ultrasonography revealed a poorly marginated heterogeneous lesion of 19 x $17 \times 21 \mathrm{~mm}$ (L x AP x T) size on right side of the vaginal vault region. It indented urinary bladder wall locally with no obvious abnormal internal vascularity. The lesion was most likely suggestive of scar endometriosis, with possibility of malignancy to be remote. Review of slides and blocks of the hysterectomy specimen showed secretory phase endometrium papillary cystic cervicitis.

Patient was taken for laparoscopic exploration that showed a $2 \times 2 \mathrm{~cm}$ nodule at the vault centrally. Cystoscopy performed showed normal bladder. The vault nodule was separated along with another Recto-ureteric nodule and sent for frozen section. Frozen section report revealed vaginal endometriosis with no evidence of malignancy. The patient did not have any recurrence in the first year follow-up.

\section{Case 3}

A 34 year old female P1L1 came with complain of haematuria at time of last menses and severe dysmenorrhea since 1 year. She had one child delivered vaginally 12 years back. Before presenting at our outpatient department, she had shown to urologist. Ultrasonography done showed bladder to be inadequately full with focal echogenic mass of $4 \times 3 \mathrm{~cm}$ with $2 \mathrm{~cm}$ thickness in posterior wall of bladder. Uterus was bulky with thickened anterior wall due to adenomyosis with 2.7 x $2 \mathrm{~cm}$ fibriod in anterior wall of uterus. MRI Pelvis showed a large altered signal intensity multiloclated space occupying lesion in bladder with muscle and perivesical fat invasion measuring 46 × $39 \mathrm{~mm}$ size in axial plane with cranio-caudal extension of $44 \mathrm{~mm}$, suggestive of urinary bladder soft tissue mass lesion. There was loss of fat plane between the anterior wall of uterus and above described mass lesion with invasion of the anterior myometrium of the uterus. There was no rectum involvement, no enlarged lymph nodes and no ascites.

She underwent biopsy of the bladder lesion, cervix and endometrium. Report showed chronic cervicitis, and secretory endometrium. The bladder biopsy specimen showed Von Bruno nest and cystitis cystica et glandularis with marked recent and old haemorrhages with no evidence of malignancy and likely to be Endometriosis. Urine examination showed 30-35 pus cells, 2-3 RBC's, 12-15 epithelial cells.

Patient was taken for laparoscopy. Cystoscopy showed bladder endometrioma, right ureter was identified and left ureter was embedded in the growth. Ureteric catheter was inserted through right ureter. Intra-operative findings revealed bladder to be densely adherent to the anterior uterine surface with free fluid in POD and endometriotic 
recto-vaginal nodule identified along left utero-sacral ligament. Incision was kept at the dome of bladder and endometriotic nodule excision was started taking care that right ureteric opening and trigone are not injured. Left ureteric opening is identified embedded in the growth but had free spill of urine. Sparing that, the entire endometriotic nodule is excised. Rectovaginal nodule is also excised. After that total laparoscopic hysterectomy was done.

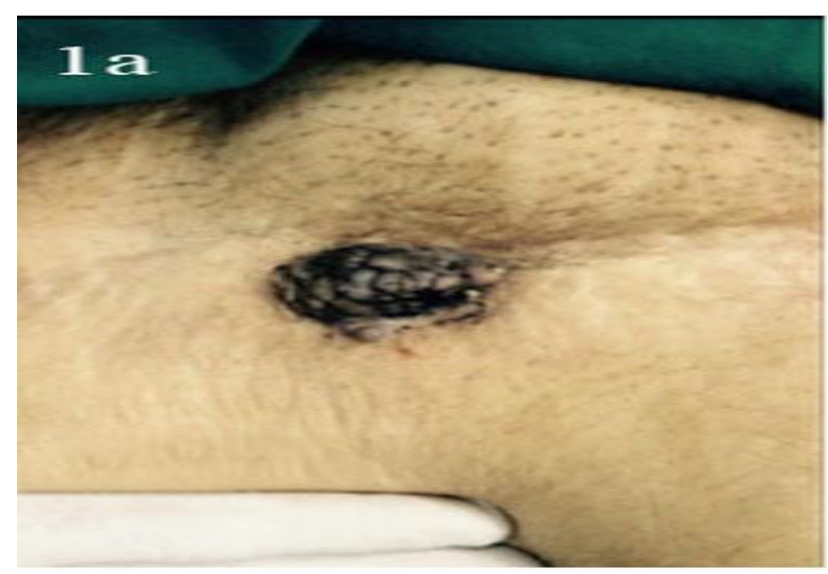

Figure 1(A): Endometriosis at left angle of caesarean scar.

Histopathological report showed chronic cervicitis with proliferative endometrium adenomyosis features. The bladder and the rectovaginal nodule showed features of endometriosis. The intra-uterine Foley's catheter was kept for 21 days. Follow up ultrasonography that was done at day 7 and at 6 month interval was normal.

\section{Case 4}

A 37 year old female, married since 12 years P1L1 had come with complain of pain in right side of abdomen, initially cramp like followed by dull aching in nature, moderate intensity, relieved partially by pain medications since 2-3 days. Patient had irregular menses with dysmenorrhea and was taking treatment for secondary infertility. She had one child alive and well through LSCS 10 years back. In 2014, 2 years back she had undergone laparoscopic partial endometriotic cyst wall excision in another private setup. Her past history was not significant. On examination per abdomen there was tenderness, more on left side than on right side, but no guarding and rigidity. On per speculum examination cervix and vagina were healthy and per vaginally uterus was normal size, fixed, retroverted. In both the fornices fullness was present. Uterine movements were tender. Vitally she was stable and systemic examination revealed no abnormality. CA-125 was found to be highly elevated i.e. $617.20 \mathrm{u} / \mathrm{ml}$. CT scan showed $8 \times 7 \times 6 \mathrm{~cm}$ thick walled non-enhancing endometriotic cyst in right adnexa causing right sided hydroureter and hydronephrosis and another $5 \times 5.5 \times 5 \mathrm{~cm}$ non enhancing endometriotic cyst in left adnexa. She was admitted and all investigations were done.

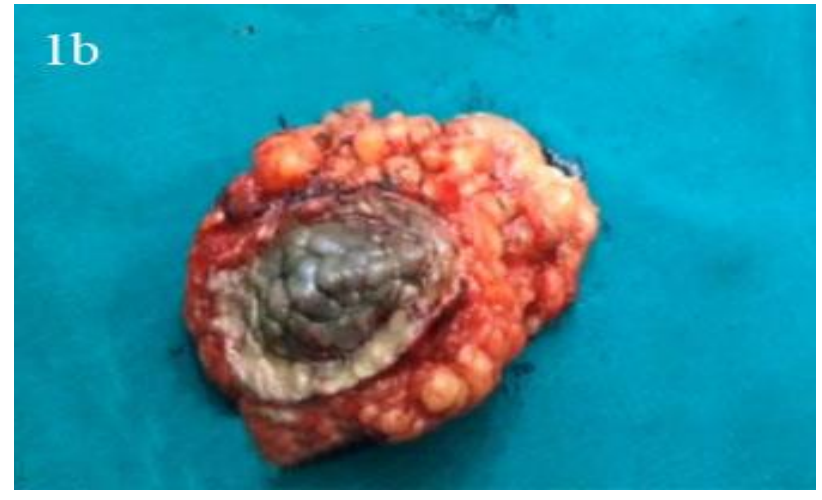

Figure 1(B): Surgical specimen of Endometriotic nodule excised with $2 \mathrm{~cm}$ margin.

She was taken for laparoscopic exploration. Intraoperatively there were large endometriotic cysts on both sides. Posterior surface of uterus was adherent to colon, rectum, uterosacrals and the tubes were adherent to ovaries and surrounding structures. On puncturing right side cyst, chocolate coloured fluid was aspirated while on left side pus like yellow coloured material was aspirated which was sent for culture.

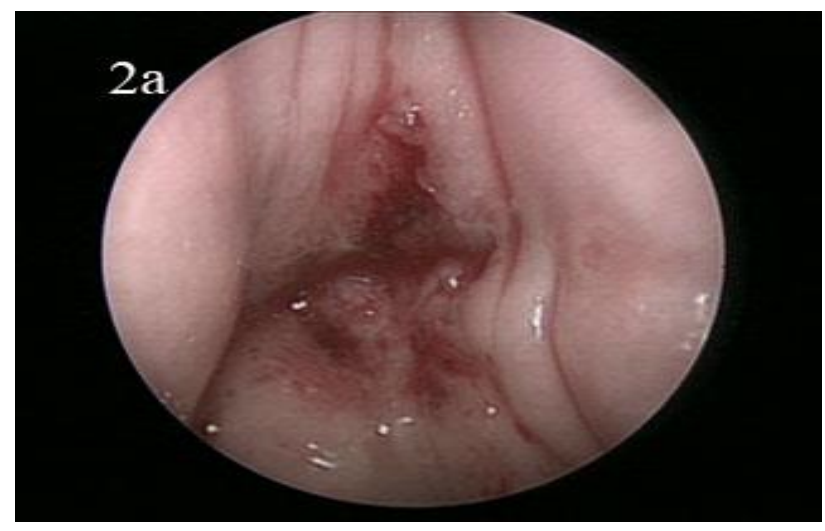

Figure 2(A): Hysteroscopy view of vault endometriosis.

Cyst wall on both side were separated from ovary. By blunt dissection and with help of harmonic scalpel ureter was exposed. Its proximal part showed hydroureter whereas the distal part was extremely fibrosed with loss of peristaltic function. The endometriotic nodule encasing ureter was removed and recto-vaginal endometriotic nodule from POD was also removed. DJ stent was passed through right ureter through cystoscopy. Histopathological report showed endometriotic cyst and culture report of yellow coloured fluid was negative for any organism. She was discharge on day 3 and was given Inj leuprolide $11.25 \mathrm{mg}$ i.m at time of discharge. 


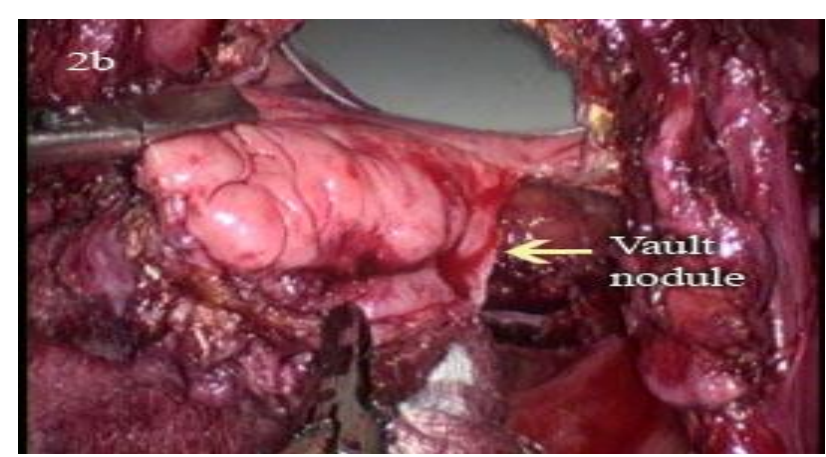

Figure 2(B): Laparoscopic view of endometriotic nodule in the vault.

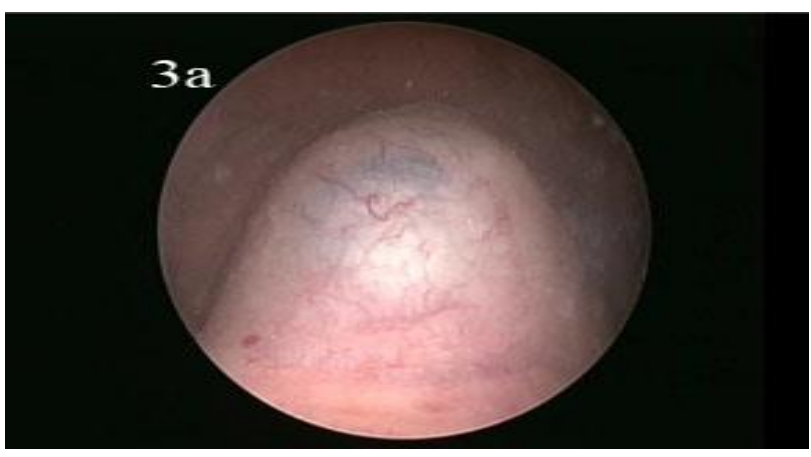

Figure 3(A): Cystoscopy view of bluish endometriotic nodule.

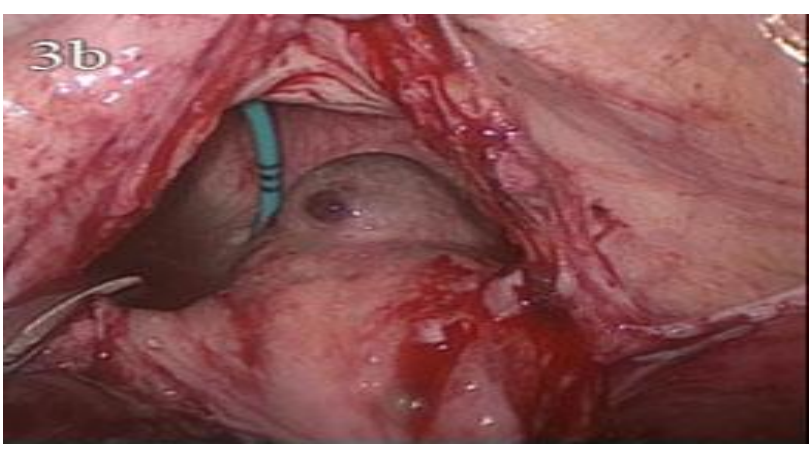

Figure 3(B): Laparoscopic view of endometriotic nodule in the bladder with DJ stent in situ.

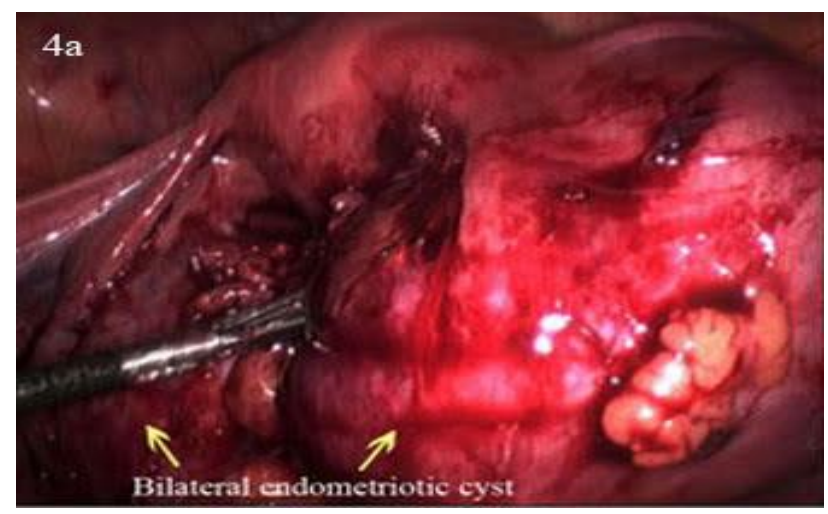

Figure 4(A): Bilateral endometriotic chocolate cyst.

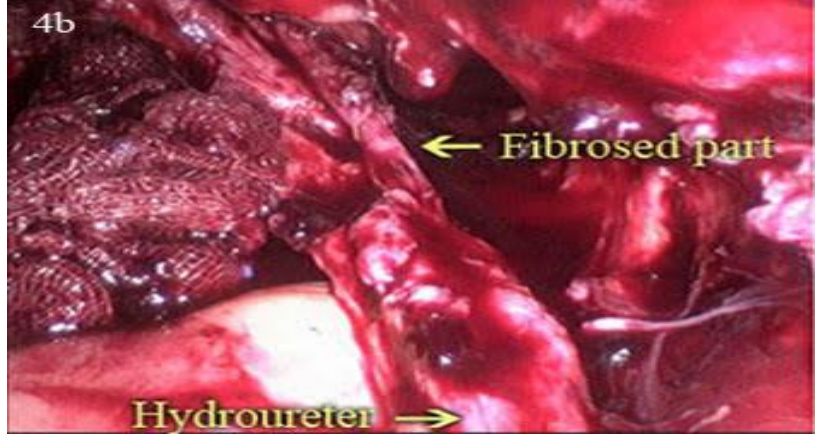

Figure 4(B): Fibrosed distal part of ureter with proximal hydroureter.

\section{DISCUSSION}

There are two classical, well-differentiated endometriosis entities in both clinical manifestations and etiopathogenesis: (1) Adenomyosis or internal endometriosis and (2) external endometriosis or simply endometriosis. (1) Adenomyosis or internal endometriosis occurs when ectopic endometrial foci infiltrate the outer muscular walls of the uterus. Adenomyoma is an area of adenomyosis that is encapsulated in the myometrial tissue. We know today that some of these masses can be another type of pathology i.e. accessory and cavitated uterine masses (ACUM) ${ }^{8,9}$ (2) External endometriosis or simply endometriosis is present when the endometrial ectopic foci are located anywhere within the pelvis (ovary, pouch of Douglas, uterosacral ligaments, rectovaginal septum, and vesicouterine pouch), abdominal cavity (bowel, omentum), or outside (lungs, brain). However, many authors refer to deep endometriosis as "adenomyosis," that is, those foci located in the rectovaginal septum or infiltrating the pouch of Douglas or the bowel (DIE-Deep Infiltrating Endometriosis). ${ }^{10,11}$ These nodules, composed of glands and scarce stroma, are surrounded by hyperplastic smooth muscle cells and are functionally capable of responding to exogenous, endogenous, or local hormonal stimuli and cause severe clinical symptoms.

The theories that were initially proposed for endometriosis were reviewed by Ridley ${ }^{12}$ and grouped into three categories (1) transplantation, (2) coelomic metaplasia, and (3) metaplasia induced by factors released into the peritoneal cavity. Therefore, there are actually two theories (transplantation and metaplasia) to which hypotheses of ectopic Mullerian rests must be added. Sampson's transplantation and implantation theory (also called the theory of retrograde menstruation) is the most widely accepted theory for the formation of ectopic endometrium in endometriosis because it may explain nearly all cases. This theory suggests that the origin of endometriosis is due to the propagation and attachment of eutopic endometrial cells (endometrium) outside the uterus, in a continuous manner (adenomyosis), through the fallopian tubes (pelvic endometriosis), or by lymphatic, hematogenous or 
mechanical dissemination (perineum or laparotomic scars).

These theories (metaplasia, Mullerian rests, and retrograde menstruation) appear to be contradictory. According to the first two hypotheses, endometriosis originates in the peritoneal serosa or from structures derived from the Mullerian ducts, whereas according to the retrograde menstruation theory, its origin is due to transplantation and implantation of uterine endometrial cells. However, the development of metaplasia or growth of Mullerian rests may be triggered due to menstrual debris that reaches the pelvic peritoneum through retrograde menstruation. Thus, these theories could be considered complementary.

Scar endometriosis is most commonly seen following surgical procedures on uterus and fallopian tubes and the incidence has been estimated to be only $0.03 \%$ to $0.15 \%$ of all cases of endometriosis. ${ }^{13,14}$ The term scar endometriosis is used for well-marked fibrous tissue, with thick chocolate-like liquid areas, and is located anywhere in the surgical scar. ${ }^{15}$ One study has reported scar endometriosis at surgical site is most commonly seen after the operation of cesarean section followed by episiotomy, hysterotomy, hysterectomy and laparotomy. ${ }^{16}$

The development of the mass of ectopic endometrial tissue in the surgical scar is most likely explained by dissemination of the endometrium or from pre-existing intraperitoneal endometriosis. ${ }^{17}$ In this patient's case there was no known history of endometriosis so it is likely that seeding of endometrial tissue during the caesarean section caused this. Direct mechanical implantation seems to be the most plausible theory for explaining scar endometriosis. During caesarean section, endometrial tissue might be seeded into the wound, and under estrogen influence these cells proliferates. ${ }^{18}$ The decidua in the early period of pregnancy has more pleuripotential capabilities and can result in cellular replication producing endometriosis thus explaining the higher incidence of endometriosis seen after hysterotomy done for mid-trimester abortions. ${ }^{19}$ Incidence of scar endometriosis following hysterotomy is $1.08 \%-2 \%$ whereas after caesarean section the incidence is $0.03 \%$ $0.4 \% .^{20}$

Our case of vault endometriosis is peculiar as patient presented with atypical symptoms and there was no prior history of endometriosis as suggested by the Histopathological report of primary surgery. One possibility is that vaginal lesion must have developed from the endometrial fragment of the uterine cavity implanted at time of primary surgery. However presence of recto-ureteric nodule indicates the presence of endometriosis at the time of primary surgery itself that may have gone unrecognized and led to development of scar endometriosis at vaginal vault by extension in posthysterectomy period. This also signifies a specialized susceptibility, either congenital or acquired to
Endometriosis rendering implantation more likely to occur. $^{21}$

Judd $^{22}$ described the first case of endometriosis affecting the bladder in 1921. Muller first described endometriosis on cystoscopy in 1927, who found an angiomatous bladder lesion which was confirmed as endometriosis by histologic study. ${ }^{23}$ However, it was Ottaw who made the first preoperative endoscopic diagnosis in 1929. Urinary tract disease is thought to occur in only $1 \%$ of cases, of which $84 \%$ involve the bladder. ${ }^{24}$ Three leading theories explain the origin of bladder endometriosis: (1) It develops from Mullerian remnants in the vesicouterine/vesicovaginal septum. (2) It is in fact an extension of an adenomyotic nodule of the anterior uterine wall. (3) It results from implantation of regurgitated endometrium. ${ }^{25}$ Broadly speaking, vesical endometriosis has 2 different causes: a primary and a secondary form. The primary form is a spontaneously occurring manifestation of a generalized pelvic disease. The secondary form is iatrogenic, occurring after pelvic surgery, such as caesarean delivery or hysterectomy. ${ }^{26,27}$ After iatrogenic dissemination, growth of ectopic endometrium is usually limited to the bladder wall. In our case absence of any history of any previous surgery and presence of adenomyosis in anterior uterine wall points towards the fact that endometriosis must have developed as an extension of the adenomyosis present in anterior uterine wall. Patients with bladder endometriosis often present frequency, dyspareunia, dysuria or cyclical hematuria as seen in our case. ${ }^{28,29}$ Presentation similar to cystitis may often delay the diagnosis. ${ }^{30}$

The ratio of bladder-to-ureteral-to-renal involvement is 40:5:1. ${ }^{31,32}$ Ureteric endometriosis, as sole manifestation of the disease was first described in the literature in $1956 .{ }^{33}$ It is rare and may result in a high rate of renal loss before it is recognized. There are estimates that $30 \%$ or nearly a 100,000 women with ureteral endometriosis will have $25 \%$ to $50 \%$ loss of nephrons at time of diagnosis of ureteral endometriosis, and an unknown number will then have loss of the kidney. ${ }^{34}$ The ureter is most commonly affected in the distal third and more on the left side $(63 \%)$ than on right. It may be extrinsic in $70-80 \%$ of cases, causing progressive stenosis of the ureter. The intrinsic form subverts the muscular layer or the ureteral mucosa, always also involving the advential coat and periureteral tissues. Urinary endometriosis has a broad spectrum of symptoms involving abdominal or flank pain, renal colic, hematuria, cyclical dysuria, urgency, frequency and suprapubic pain. This case is unique in its own due to its recurrence after undergoing surgery for the same two years back and the unusual localization and minimal symptoms which developed recently in form of abdominal pain inspite of wide spread of the disease leading to hydroureteronephrosis and possible infertility. The raised levels of CA-125 must be due to large surface areas of endometriomas and endometriosis induced peritoneal inflammation. ${ }^{35}$ Infertile women are 6 to 8 times more likely to have endometriosis than fertile 
women. ${ }^{36}$ Many mechanisms like distorted pelvic anatomy, endocrine and ovulatory abnormalities, altered peritoneal function, and altered hormonal and cellmediated functions in the endometrium have been proposed to explain infertility in endometriosis cases but no definite conclusion has been reached.

In practice, it is not easy to diagnose endometriosis due to its variability in symptomatology and its anatomic and clinical parallelism. Experience with the disease in a medical practice assists in establishing a firm diagnosis more than what has traditionally been thought. In these patients, correct diagnosis depends on careful examination, right questioning and obviously taking endometriosis into consideration. A good surgical and gynecological history, as well as a thorough examination with appropriate imaging techniques (ultrasound, CT or MRI) will usually lead to a correct diagnosis. Endometriosis should be considered in any woman of reproductive age who has chronic pelvic pain (non-cyclic abdominal and pelvic pain of at least 6 months' duration). ${ }^{37}$ The most common symptoms are dysmenorrhea, dyspareunia, and lower back pain that worsens during menses. ${ }^{38}$ Atypical presentations suggesting more significant disease involvement include cyclic leg pain or sciatica (nerve involvement) and cyclic dyspnea secondary to catamenial pneumothorax. Gastrointestinal and genitourinary involvement may be indicated by the presence of symptoms such as constipation, diarrhoea, hematochezia, dyschezia, dysuria, cyclic urinary frequency, urgency or haematuria that may guide clinical investigations and imaging.

We must consider two important signs: deep dyspareunia and nodules in the pouch of Douglas. Physical examination should be performed during early menses, when implants are likely to be largest and most tender. In external locations, such as a laparotomic scar or the perineum or umbilicus, endometriosis can appear as a painful, hard, blue-black, or brown nodule that grows during menstruation. In the cervix, endometriosis may be present as velvety red lesions during menstrual cycles that may darken to a deep purple colour during bleeding. ${ }^{39}$ The physician should palpate for a fixed, retroverted uterus, adnexal and uterine tenderness, pelvic masses, or nodularity along the uterosacral ligaments. A rectovaginal examination is required to identify uterosacral, cul-de-sac, or septal nodules. An enlarged, tender, cystic adnexal mass may suggest an endometrioma. Although occurrence of vault site endometriosis is extremely rare, it must be considered in delayed bleeding occurring in a post-hysterectomy patient when other diagnoses have been excluded. The presence of cyclic pain with or without bleeding in an incisional mass associated with a caesarean section scar is almost pathognomonic for the condition.

Imaging has limited utility in the diagnosis of endometriosis, as it lacks adequate resolution to identify adhesions or superficial peritoneal implants.
Ultrasound is a readily available, inexpensive and most commonly used tool for the diagnosis of large endometriosis lesions. Transvaginal ultrasound can help diagnose endometriomas, bladder lesions, and deep nodules like those in the rectovaginal septum. Lesions identifiable on ultrasound include hypoechogenic linear thickening or nodules/masses with or without regular contours and messy internal echoes.

MRI may help guide surgical approaches for patients with suspected endometriosis, especially for ultrasonographically indeterminate deep infiltrating endometriosis, rectosigmoid lesions, endometriosis of the bladder and other unusual sites of presentation. MRI much better assesses infiltration of abdominal wall muscles and subcutaneous tissues. MRI has very high spatial resolution, which enables accurate detection of very small hemorrhagic lesions. As CT of the pelvis does not visualize pelvic organs well, it is not useful in the diagnosis of endometriosis but can be used to detect ureteral involvement and possible renal insufficiency. ${ }^{40}$ Cystoscopy is able to visualize the endometriosis foci only when present on bladder mucosal surface, but is unable to define the extent of endometriosis lesion, which can be better detected by TV sonography.

Fine-needle aspiration cytology (FNAC) was reported in some studies for confirming the diagnosis. However chances of reimplantation of potential malignancies and recurrence of endometriosis by seeding of needle tract must be known. Histology is the hallmark of diagnosis. Diagnosis requires the presence of two or more of these histologic features: endometrial epithelium, endometrial glands, endometrial stroma, and hemosiderin-laden macrophages. ${ }^{41}$ Generally, diagnosis is easy with a microscopic examination of a standard hematoxylin and eosin-stained slide. Furthermore, the cytologist experience must be the important point to clarify diagnosis and to exclude malignancy.

However, most women with endometriosis have normal pelvic findings, and laparoscopy is necessary for definitive diagnosis. At laparoscopy, endometriosis may be visualized as peritoneal implants, peritoneal windows, endometriomas, and deep infiltrating nodules of endometriosis that may each be associated with adhesions. Endometriosis typically appears as superficial "powder burn" or "gunshot" lesions on the ovaries, serosal surfaces and peritoneum black, dark-brown, or bluish puckered lesions, nodules or small cysts containing old haemorrhage surrounded by a variable extent of fibrosis. Atypical or 'subtle' lesions are also common, including red implants (petechial, vesicular, polypoid, hemorrhagic, red flame-like) and serous or clear vesicles. Other appearances include white plaques or scarring and yellowish brown peritoneal discoloration of the peritoneum. Endometriomas usually contain thick fluid like tar; such cysts are often densely adherent to the peritoneum of the ovarian fossa, posterior surface of uterus and the surrounding fibrosis may involve the tubes 
and bowel. Deeply infiltrating endometriotic nodules extend $>5 \mathrm{~mm}$ beneath the peritoneum and may involve the uterosacral ligaments, vagina, bowel, bladder, or ureters. The depth of infiltration is related to the type and severity of symptoms. ${ }^{42}$ Medical therapy with danazol, progesterone, Gn-Rh agonists, produces only partial recovery, and mostly recurrence occurs after cessation of the treatment with extreme side effects. ${ }^{43}$

Laparoscopy is the preferred route for surgical management of endometriosis and is considered to be gold standard, irrespective of severity, owing to the greater visualization through a magnified view allowing close examination and visualization of endometriotic implants and the quicker patient recovery and return to normal activity when compared with laparotomy. ${ }^{44}$

Local wide excision with $1 \mathrm{~cm}$ margin to ensure complete removal of the disease is considered to be accurate treatment of choice for scar endometriosis and also for recurrent lesions and allays the remote concern for malignant transformation. As expected, the larger and lesions deeper to the muscle or the fasciae are more difficult to excise completely and may require the placement of a synthetic mesh, or transfer of tissue for closure. $^{45}$ The incidence of concomitant pelvic endometriosis with scar endometriosis has been reported to be from $14.3 \%$ to $26 \%$. Ideally, all patients must be examined for concomitant pelvic endometriosis and at this point postoperative follow-up should be done for a couple of years and the patient should be under the observation of the gynecologist.

In case of urogenital endometriosis the choice of treatment depends on different factors, such as the numbers and size of the lesion, its location, and its severity. Segmented resection, open and laparoscopic partial cystectomy or transurethral resection of the bladder mass, with ureterolysis (if necessary) may be performed. Hormonal treatment can be started in those wishing to preserve fertility. However, experienced laparoscopists, familiar with endometriosis, can also treat urogenital endometriosis safely and effectively using excision. Endoscopic resection of the lesions is currently considered the treatment of choice when possible. ${ }^{46}$

In ureteral endometriosis treatment is generally aimed at relieving symptoms and ureteral obstruction, rescuing the involved kidney and prevention of recurrence. Surgical interventions for relief of obstructive uropathy include ureterolysis, ureteroureter-ostomy, distal ureterectomy, and ureteral reimplantation or interposition of ileal segment between the ureter and bladder. All surgical approaches begin with identification of the ureter. Ureterolysis is carried out starting proximal to the diseased area, at a level of healthy tissue unaffected by endometriosis proceeding down to the level of damage. A decision is made at this point, based on extent and localization of disease, as to whether ureterolysis will be adequate to relieve obstruction. Ureterolysis is often acceptable in cases of extrinsic, nonobstructive disease but is contraindicated in intrinsic one due to chance of recurrence and stenosis. ${ }^{47}$ In our case after resection of the ureteric nodule the proximal hydroureter decreased in size and DJ stent was passed easily, so conservative approach was applied. Treatment of patients having intrinsic UE includes resection of the ureter and end to end anastomosis, but changes according to the location and severity.

Medical and surgical treatments for endometriosis have different effects on a woman's chances of conception, either spontaneously or via assisted reproductive technologies (ART). Surgery at any stage of endometriosis enhances the chances of natural conception. Criteria for non-removal of endometriomas are: bilateral cysts, history of past surgery, and altered ovarian reserve. Exceptions to the rule of no surgery before ART as surgery can alter ovarian function are pain, hydrosalpinges, very large endometriomas, rapid growth or suspicious features noted on ultrasound. Stripping the endometriotic cyst wall from the ovary is advised because of the high recurrence rate after laparoscopic drainage of an endometrioma without removal. Needless to say, if endometrioma resection is performed, it is critical to proceed conservatively and to minimize compromise of ovarian blood supply and preserve normal ovarian tissue. ${ }^{48}$ The goal of laparoscopic surgery is to resect endometriotic lesions as much as possible, restore normal anatomy with adhesiolysis, and optimize ovarian and tubal preservation and integrity with use of the principles of microsurgery (magnification, diligent hemostasis, reduced fulguration, avoidance of tissue drying, and limited use of sutures) and is the preferred surgical route of approach. Medical management though contraceptive in nature but timely pre-treatment e.g., 3-6 months of gonadotropin-releasing hormone analogues can improve the outcome of ART. When age, ovarian reserve, and male and tubal status permit, surgery should be considered immediately so that time is dedicated to attempts to conceive naturally. In other cases, the preference is for administration of gonadotropin-releasing hormone analogues before ART and no surgery beforehand. Weighing up the relative advantages of surgery, medical treatment and ART are the foundations for a global approach to infertility associated with endometriosis. ${ }^{49,50}$ The respective advantages of surgery, medical treatment, and ART intertwine complexly in women with these disorders. This intricate medley mandates a global approach to optimize every option.

\section{CONCLUSION}

Emery Wilson stated that endometriosis "remains a riddle wrapped in a mystery inside an enigma". Endometriosis is a progressive and elusive disease. The key to treatment rests with recognition of symptoms and directed intervention. One should have high index of suspicion of scar endometriosis when a woman presents with a painful 
swelling in the abdominal scar with cyclical pain, especially with a history of previous gynaecological or obstetrical surgery. A multidisciplinary team approach, including a skilled, advanced laparoscopic gynaecologist, urologist, and colorectal surgeon play key roles in the successful treatment of extensive disease. Every effort should be made to treat endometriosis actively at first laparoscopy with aim of complete removal of all visible lesions. The limiting factors in effectively treating extensive disease are the skill of the surgeon and the availability of proper instrumentation. In case of infertility with moderate to severe endometriosis, there is good evidence that in experienced hands laparoscopic surgery has a lot to offer in infertile women. Inspite of being technically demanding, surgical treatment is patient rewarding in enhancing fertility. Good technique and proper care during caesarean section like discarding the used sponge immediately after cleaning the uterine cavity and not reusing the suture material used for uterus while closing abdominal wall may help in preventing endometriosis. To prevent the occurrence of scar endometriosis it has been suggested that at the end of surgery especially on uterus and tubes, the abdominal wall wound should be cleaned thoroughly and irrigated vigorously with high jet solution before closure.

\section{Funding: No funding sources}

Conflict of interest: None declared

Ethical approval: Not required

\section{REFERENCES}

1. Endometriosis. In Fritz MA, Speroff L Clinical gynecologic Endocrinology and Infertility.8th ed.Wolters Kluwer: Lippincott Williams and Wilkins; 2011:1221-1248.

2. Acién P, Velasco I. Endometriosis: A Disease That Remains Enigmatic. ISRN Obstetrics and Gynecology. 2013; Article ID 242149, 12 pages.

3. Jubanyik KJ, Comite F. Extrapelvic endometriosis. Obstet Gynecol Clin North Am. 1997;24:411-40.

4. Heaps JM, Nieberg RK, Berek JS. Malignant neoplasms arising in endometriosis. Obstet Gynecol. 1990;75:1023-8.

5. Teng CC, Yang HM, Chen KF, Yang CJ, Chen LS, Kuo CL. Abdominal wall endometriosis: an overlooked but possibly preventable complication. Taiwan J Obstet Gynecol. 2008;47:42-8.

6. Endometriosis. In: Fritz MA, Speroff L Clinical gynecologic Endocrinology and Infertility.8th ed.Wolters Kluwer: Lippincott Williams and Wilkins; 2011:1221-1248.

7. Sengul I, Sengul D, Kahyaoglu S, Kahyaoglu I. Incisional endometriosis: A report of 3 cases. Can J Surg. 2009;52:444-5.

8. Acien P, Acien M. The cavitated accessory uterine mass. A Mullerian anomaly in women with an otherwise normal uterus. Obstetrics and Gynecology. 2010;116:1101-9.
9. Acien P, Bataller A. New cases of accessory and cavitated uterine masses (ACUM): a significant cause of severe dysmenorrhea and recurrent pelvic pain in young women. Human Reproduction. 2012;27(3):683-94.

10. Koninckx PR, Martin DC. Deep endometriosis: a con- sequence of infiltration or retraction or possibly adenomyosis externa? Fertility and Sterility. 1992;58(5):924-8.

11. Koninckx PR, Martin D. Treatment of deeply infiltrating endometriosis. Current Opinion in Obstetrics and Gynecology. 1994;6(3):231-41.

12. Ridley JH. The histogenesis of endometriosis: a review of facts and fancies. Obstetrical and Gynecological Survey. 1968;23(1).

13. Francica G, Giardiello C, Angelone G, Cristiano S, Finelli R, Tramontano G. Abdominal wall endometriosis near cesarean delivery scars. J Ultrasound Med. 2003;22:1041-7.

14. Kaloo P, Reid G, Wong F. Caesarean section scar endometriosis: Two cases of recurrent disease and a literature review. Aust NZ J Obstet Gynaecol. 2002;42:218-20.

15. Meirelles M, Losano R, Viana AT. Scar endometriosis: study of 14 cases. Arq Med Hosp Fac Cienc Med Santa Casa Sao Paulo. 2005;50(3):92-6.

16. Blanco RG, Parithivel VS, Shah AK, Gumbs MA, Schein M, Gerst PH. Abdominal wall endometriomas. Am J Surg. 2003;185:596-8.

17. Zhao X, Lang J, Leng J, Sun D, Zhu L. Abdominal Wall Endometriomas. International Journal of Gynecology and Obstetrics. 2005;90:218-22.

18. Gunes M, Kayikcioglu F. Incisional endometriosis after cesarean section, episiotomy and other gynecologic procedures. Journal of Obstetrics and Gynaecology Research. 2005;31(5):471-5.

19. Scott RB, Telinde RW. Clinical external endometriosis; probable viability of menstrually shed fragments of endometrium. Obstetrics and gynecology. 1954;4(5):502-10.

20. Chatterjee SK. Scar endometriosis: A Clinicopathological study of 17 cases. Obstet Gynecol. 1980;56:81-84.

21. Green TH Jr, Meigs JV. Pseudo menstruation from post hysterectomy vaginal vault endometriosis. Obstet Gynecol. 1954;4:622-34.

22. Judd ES. Adenomyoma presenting as a tumor of the bladder. Surg Clin North Am. 1920;1:1271-3.

23. Muller J. Endometrioide adenomatose (denomyosis) and cystadenomatose Arch F Klin Chir. 1927;145:394-434.

24. Umaria N, Olliff JF. MRI appearances of bladder endometriosis. The British J Radiol. 2000;73:733-6.

25. Vercellini P, Frontino G, Pisacreta A, De Giorgi O, Cattaneo M, Crosignani PG. The pathogenesis of bladder endometriosis. Am J Obstet Gynecol. 2002;187:538-42.

26. Agarwal N, Kriplani A. Intramural bladder endometriosis after caesarean section: diagnostic and therapeutic aspects. J Gynecol Surg. 2002;18:69 -73. 
27. Mettler L. Manual of New Hysterectomy Techniques. New Delhi, India: Jaypee Brothers Medical Publishers (P) Ltd; 2007.

28. Chen WM, Yang CR. Vesical endometriosis: a case report. JTUA. 2000;14(4):183-6.

29. Westney OL, Amundsen CL, McGuire EJ. Bladder endometriosis: conservative management. J Urol. 2000;163:1814-7.

30. Bogart LM, Berry SH. Symptoms of interstitial cystitis, painful bladder syndrome and similar diseases in women: a systematic review. J Urol. 2007;177(2):450-6.

31. Stillwell TJ, Kramer SA, Lee RA. Endometriosis of ureter. Urology. 1986;28:81-5.

32. Jubanyik KJ, Comite F. Extrapelvic endometriosis. Obstet Gynecol Clin North Am. 1997;24:411-40.

33. Leonhartsberger N, Zelger B. Intrinsic Endometriosis of Ureter and Bladder in Young Women without Gynaecological Symptoms. Urol Int. 2008;80:222-4.

34. Horn LC, Do Minh M, Stolzenburg JU. Intrinsic form of ureteral endometriosis causing ureteral obstruction and partial loss of kidney function. Urol Int. 2004;73:181-4

35. Rani AK, Kapoor D. Ruptured ovarian endometrioma with an extreme rise in serum CA 125 level - A case report: Ovarian endometrioma with very high CA-125 level. Gynecologic Oncology Case Reports. 2012;2(3):100-1.

36. Verkauf BS. The incidence, symptoms, and signs of endometriosis infertile and infertile women. J Fla Med Assoc. 1987;74(9):671-5.

37. Milburn A, Reiter RC, Rhomberg AT. Multidisciplinary approach to chronic pelvic pain. Obstet Gynecol Clin North Am. 1993;20:643-61.

38. American College of Obstetricians and Gynecologists. Washington DC; Endometriosis. ACOG technical bulletin no. 184. ACOG; 1993.

39. Acién P, Velasco I. Endometriosis: A Disease That Remains Enigmatic. ISRN Obstetrics and Gynecology, 2013; Article ID 242149, 12 pages.

40. Hsu AL, Khachikyan I, Stratton P. Invasive and noninvasive methods for the diagnosis of endometriosis.
Clinical obstetrics and gynecology. 2010;53(2):4139.

41. Medical Management of Endometriosis. ACOG Practice Bulletin \#11. 1999

42. Kennedy S, Bergqvist A, Chapron C, D'Hooghe T, Dunselman G, Greb R, et al. ESHRE guideline for the diagnosis and treatment of endometriosis. Hum Reprod. 2005;20:2698-704.

43. Rivlin ME, Das SK, Patel RB, Meeks GR. Leuprolide acetate in the management of cesarean scar endometriosis. Obstet Gynecol. 1995;85:838-9.

44. Crosignani PG, Vercellini P, Biffignandi F, Costantini W, Cortesi I, Imparato E, et al. Laparoscopy versus laparotomy in conservative surgical treatment for severe endometriosis. Fertil Steril. 1996;66:706-11.

45. Patterson GK, Winburn GB. Abdominal wall endometriomas: Report of eight cases. Am J Surg. 1999;65:36-9.

46. Maccagnano C, Pellucchi F, Rocchini L, Ghezzi M, Scattoni V, Montorsi F, et al. Diagnosis and Treatment of Bladder Endometriosis: State of the Art. Urol Int. 2012;89:249-58.

47. Nezhat C, Paka C, Gomaa M, Schipper E. Silent Loss of Kidney Secondary to Ureteral Endometriosis. JSLS: Journal of the Society of Laparoendoscopic Surgeons. 2012;16(3):451-5.

48. Tang Y, Chen SL. Ovarian damage after laparoscopic endometrioma excision might be related to the size of cyst. Fertility and Sterility. 2013;100(2):464-9.

49. de Ziegler. Endometriosis and infertility: pathophysiology and management. The Lancet. 2012;376(9742):730-8.

50. Chatterjee SK. Scar endometriosis: A clinicopathologic study of 17 cases. Obstet Gynecol. 1980;56:81-4.

Cite this article as: Limbachiya DJ, Agrawal GP. Endometriosis: an enigmatic disease with many faces. Int J Reprod Contracept Obstet Gynecol 2017;6:730-8. 